\title{
Seguridad del ácido zoledrómico en el control del dolor en el estadio Mlb del cáncer de próstata
}

\author{
Álvarez-Múgica M*, Fernández Gómez JMª*, Jalón Monzón A*, González Álvarez RC*, \\ García Rodríguez J*, Martín Benito JL*.
}

*Servicio de Urología 1. Hospital Universitario Central de Asturias. Oviedo. Asturias.

Actas Urol Esp. 2007;31(10):1100-1106

\section{RESUMEN}

SEGURIDAD DEL ÁCIDO ZOLEDRÓMICO EN EL CONTROL DEL DOLOR EN EL ESTADIO M1b DEL CÁNCER DE PRÓSTATA

Objetivos: Valorar el uso del ácido zoledrómico en el control del dolor de las metástasis óseas en el cáncer de próstata, así como estudiar su seguridad y efectos secundarios.

Material y métodos: Se incluyeron en el estudio 37 pacientes consecutivos diagnosticados de cáncer de próstata con metástasis óseas entre los años 2005 y 2006 a los que se administró una dosis de 4 mg de Zometa ${ }^{\circledR}$ mensual durante una media de 10,24 meses, recogiéndose unos datos según un protocolo establecido.

Resultados: En cuanto al control del dolor, observamos como en 8 de los 20 pacientes a los que se les administró Zometa ${ }^{\circledR}$, el control fue absoluto, en 6 de estos se consiguió un control parcialmente bueno (permitiendo una buena actividad diaria), en 4 el control fue parcialmente malo (empeorando su calidad de vida), mientras que 2 pacientes presentaron mal control del dolor. Respecto a los efectos adversos del Zometa ${ }^{\circledR}$, no observamos ningún efecto secundario en ninguno de nuestros pacientes ni durante la inyección del fármaco ni posteriormente, si bien en 2 pacientes se suspendió el tratamiento por su mal estado general.

Conclusiones: El futuro de este tratamiento reside en saber si la administración precoz de este fármaco puede prevenir o retrasar la aparición de las metástasis, (merced a su actividad antitumoral demostrada), no sólo en las debidas al cáncer de próstata si no también a metástasis óseas secundarias a otros tumores genito-urinarios.

Palabras clave: Ácido Zoledrómico. Metástasis óseas. Dolor óseo.

\section{ABSTRACT}

USE OF ZOLEDROMIC ACID ON PAIN CONTROL IN STAGES MIb OF PROSTATE CANCER

Objectives: To probe the use of Zoledromic acid in the control of the pain due to bone methastasis of a prostate cancer.

Material and method: We included in our study 37 consecutive patients diagnosed of prostate cancer who developed bone methastasis during 2005 and 2006 to whom we gave a $4 \mathrm{mg}$ dose of Zoledromic acid during a mean time of 10.24 months.

Results: We observed a complete control of the pain in 8 of the 20 patients, and partially in 6 of those patients. We did not evaluate any adverse reaction due to this treatment.

Conclusions: We still yet to know whether the administration of this treatment in the early stages of the prostate cancer could prevent or retard the appearance of bone methastasis. 
$\mathrm{E}$ cáncer de próstata $(\mathrm{CP})$ es el tumor más frecuente en varones después de los cánceres de piel y de pulmón, siendo en Estados Unidos (EEUU) el segundo después del cáncer de piel. Es el causante del $11 \%$ de las muertes por cáncer en el hombre y es la segunda causa de muerte por cáncer en varones, después del cáncer de pulmón. Cada año se diagnostica un cáncer de próstata por cada de 500.000 varones en todo el mundo y son más de 200.000 los que fallecen a causa de esta enfermedad ${ }^{1}$. En la Unión Europea, la incidencia es de unos 84.000 casos por año y causa 35000 fallecimientos anuales. Las tasas de incidencia y mortalidad son del 55 y del 22,6 por 100.000 habitantes respectivamente. Más del 95 $\%$ de los tumores primarios de la próstata son adenocarcinomas. En general, el grado de diferenciación tumoral y la anormalidad del comportamiento histológico de crecimiento están íntimamente relacionados con la probabilidad de metástasis y con la muerte por esta enfermedad ${ }^{1}$. La velocidad de crecimiento tumoral varía desde muy lenta a moderadamente rápida y algunos pacientes tienen una supervivencia prolongada incluso después de que el cáncer haya hecho metástasis en sitios distantes, por ejemplo en el tejido óseo.

En España, en el año 2002, se produjeron 5678 muertes por esta enfermedad, lo que representa una tasa de 27,6 muertes/ 100.000 varones $\mathrm{y}$ año, siendo el tercer tumor maligno en cuanto a mortalidad tras el cáncer de pulmón y el colorrectal. Desde el año 1952 al 1996 la tendencia de mortalidad por este tumor en España ha experimentado un aumento del orden del $1 \%$ anual, disminuyendo ligeramente cuando se limita el análisis al periodo de $1982-1996^{2}$.

Desde el comienzo de la década de los 90, los nuevos métodos de cribado y la disponibilidad de mejores tratamientos produjeron modificaciones notables en la incidencia de este tumor, en el momento del diagnóstico y en la mentalidad de esta afección. Los importantes progresos que se registraron en la biología molecular y en la epidemiología han aportado nuevas perspectivas en relación con la etiología y la biología celular del CP. Estos desarrollos permiten albergar la esperanza de una mejor comprensión de la enfermedad y conducirán muy probablemente a nuevas y mejores modalidades de prevención y tratamiento del $\mathrm{CP}$ en un futuro cercano ${ }^{3}$.
Una vez que el CP ha sido diagnosticado clínicamente su progresión es particularmente lenta. En los últimos 20 años, la supervivencia en los varones con $\mathrm{CP}$ a los 5 años para el conjunto de todos los estadios, ha aumentado del $67 \%$ al 98 $\%$, siendo de $84 \%$ y $56 \%$ a los 10 y 15 años, respectivamente. Después de un diagnóstico inicial de enfermedad localizada, transcurre una media de 7 años antes de que las concentraciones de antígeno prostático específico (PSA) empiecen a aumentar en los pacientes que presentan una recidiva de la enfermedad. La intervención precoz por una recidiva basada solamente en un aumento de la concentración de PSA, consiste habitualmente en la instauración de un tratamiento de deprivación androgénica (TDA). La orquidectomía, los agonistas de la hormona liberadora de la hormona luteinizante, los antiandrógenos y los estrógenos, son formas diferentes de modificar los andrógenos. Además, el TDA a largo plazo se utiliza con frecuencia en los pacientes en los que se detecta un CP metastático en ganglios linfáticos extirpados en la intervención quirúrgica y de esta forma, esos pacientes pueden tener una mediana de supervivencia de más de 10 años. El tratamiento de deprivación androgénica da lugar a una reducción efectiva de la testosterona sérica hasta valores de castración. Sin embargo, esto puede causar una disminución de la masa ósea inducida por el tratamiento del cáncer, aumentando el riesgo de fracturas ${ }^{4}$. De hecho, el TDA a largo plazo produce una pérdida ósea superior a la observada en las mujeres postmenopáusicas. Tras el diagnóstico inicial de una recidiva, el CP progresa a menudo a una enfermedad metastática avanzada, entre 3-5 años, con presencia de metástasis frecuentes, especialmente las óseas ${ }^{4}$.

La morbilidad esquelética puede ser un efecto de las metástasis óseas, que aparecen en el 65-75 $\%$ de los enfermos con un CP avanzado, pero es producida también por la pérdida ósea causada por los tratamientos de ablación androgénica ${ }^{5}$.

La localización ósea metastático más frecuente del CP es:

- Espina dorsal (74\%) mayoritariamente en región lumbar y torácica.

- Costillas (70\%).

- Pelvis (60\%).

- Fémur (44\%).

- Rodillas (41\%). 
Después del diagnóstico de metástasis óseas, los varones con $\mathrm{CP}$ tienen un rango de supervivencia de 12 a 53 meses, presentando un riesgo elevado de complicaciones esqueléticas durante ese tiempo ${ }^{5}$. En estudios clínicos de varones con CP y metástasis óseas, aproximadamente un 30$50 \%$ de los pacientes experimentaron al menos un evento relacionado con el esqueleto a lo largo de 2 años de seguimiento ${ }^{5,6}$. Estos eventos relacionados con el esqueleto causan reducciones significativas en las puntuaciones de calidad de vida de estos pacientes. Estos problemas de calidad de vida se han identificado como factores independientes en la progresión de la enferme$\operatorname{dad}^{7}$. Además, las fracturas pueden estar correlacionadas con una reducción de la supervivencia, de hecho el 10 al $20 \%$ de los varones ancianos fallecen en un plazo de 6 meses tras haber sufrido una fractura de cadera ${ }^{8}$.

Las complicaciones esqueléticas (incluyendo las fracturas patológicas, la compresión de la médula espinal y el dolor óseo debilitante que requiere radioterapia) tienen un efecto negativo sobre la calidad de vida y son una causa importante de morbilidad y de posible mortalidad en estos pacientes ${ }^{5,8}$.

La Asociación Internacional para el Estudio del Dolor, define el dolor como una experiencia sensorial y emocional desagradable, relacionada con daño tisular actual o potencial, o descrito en términos de dicho daño. El dolor originado por un proceso tumoral, puede controlarse eficazmente a través de medios relativamente sencillos hasta en un 90\% de pacientes con cáncer o antecedentes de cáncer. Desafortunadamente, el dolor relacionado con el cáncer suele ser tratado con deficiencia ${ }^{9}$. Los pacientes con cáncer avanzado experimentan múltiples síntomas concurrentes y dolor; por tanto, un manejo óptimo del dolor necesita de una evaluación sistémica de los síntomas y un manejo adecuado para obtener una calidad de vida óptima ${ }^{11}$. La Organización Mundial de la Salud (OMS) ha establecido una jerarquía de tres niveles para el control del dolor ${ }^{9}$. Esta consiste de un enfoque ascendente según la severidad del dolor. Si el dolor es ligero, se puede comenzar por recetar un analgésico del primer nivel como el acetaminofeno o un fármaco antiinflamatorio no esteroide (AINE). Se debe tener en cuenta los posibles efectos secundarios adversos, sobre todo los de tipo renal o gastrointestinal de los AINEs. Si el dolor persiste o tiende a agravarse a pesar de aumentos adecuados en las dosis, entonces se debe pasar a un analgésico de grado 2 o de grado 3. La mayor parte de los pacientes de cáncer requieren analgésicos de nivel 2 o de grado 3 . El primer nivel se puede omitir en aquellos pacientes que presentan al comienzo un dolor que va de moderado a severo en beneficio del nivel 2 o del grado 3. En cada nivel se puede considerar el uso de un fármaco o tratamiento adyuvante como la radioterapia $u$ otros fármacos en determinados pacientes. Las recomendaciones de la OMS se basan en la disponibilidad de drogas en el mundo entero y no estrictamente en la farmacología.

El ácido zoledrónico (Zometa $\left.{ }^{\circledR}\right)$, es un bisfosfonato de tercera generación, tratándose del inhibidor más potente de la actividad osteoclástica de entre todos los bisfosfonatos investigados. Estudios preclínicos ha propuesto los siguientes mecanismos de acción del ácido zoledrónico:

- Inhibición de la maduración de los osteoclastos.

- Inhibición del reclutamiento de los osteoclastos en el lugar de la resorción ósea.

- Inhibición de la función osteolítica del osteoclasto.

- Inducción de la apoptosis de osteoclastos.

- Reducción de la producción de citoquinas inductoras de osteolisis.

- Actividad antitumoral.

- Inhibición de la adhesión e invasión de las células tumorales al hueso.

- Efecto antiangiogénico.

Respecto a los efectos adversos descritos, aparecen: fatiga, anemia, mialgias, fiebre, edema en extremidades, hipocalcemia e insuficiencia renal.

\section{MATERIAL Y MÉTODOS}

Se incluyeron en el estudio 37 pacientes consecutivos diagnosticados de cáncer de próstata que presentaron metástasis óseas entre los años 2005 y 2006 a los que se administró una dosis de $4 \mathrm{mg}$ de Zometa ${ }^{\circledR}$ mensual durante una media de 10,24 meses. La administración de este fármaco fue realizada por la misma enfermera en una sala con el paciente en decúbito supino. Se diluía el 
fármaco en 50 cc de suero fisiológico y se administraba en infusión continua durante 20 minutos. Posteriormente se dejaba al paciente en esta posición durante otros 20 minutos. Asímismo, el paciente recibía una dosis diaria de $500 \mathrm{mg}$ de calcio y 400 UI de vitamina D. Se realizaron controles mensuales con bioquímica y hemograma para controles de niveles de calcio y función renal.

De todos los casos se recogió nombre y apellidos del paciente con su número de historia, año de nacimiento así como fecha del diagnóstico del cáncer de próstata. Se analizaron las variables que se mencionan a continuación:

1. Edad al diagnóstico.

2. Gleason al diagnóstico: $<7,7$ ó $>7$.

3. Estadio TNM al diagnóstico.

4. PSA al diagnóstico.

5. Tipo de metástasis al diagnóstico: Óseas, Viscerales.

6. Número de metástasis óseas: $<70 \geq 7$.

7. Tratamiento inicial: observación, prostatectomía radical, braquiterapia, radioterapia externa, hormonoterapia, otros.

8. Meses hasta hormonoresistencia.

9. PSA en el momento de hormonoresistencia.

10. Tratamiento de la hormonoresistencia.

11. Dolor óseo.

12. EREs (Eventos esqueléticos)

13. Tratamiento del dolor

14. Motivo de inicio de Zometa: paciente con diagnostico de metástasis óseas asintomático o con dolor por las metástasis óseas.

15. Número de dosis de Zometa

16. Efectos secundarios del Zometa

17. Suspensión del Zometa: si, no.

18. Meses de seguimiento desde inicio del Zometa.
19. Situación actual: vivo con enfermedad estable, vivo con enfermedad progresiva, muerto por el tumor, muerto por otra causa.

20. Control del dolor: si parcial, si total, no. (mediante un cuestionario que rellenaban los pacientes cuando acudían a consulta).

Los resultados fueron almacenados en una base de datos y procesados mediante el programa SPSS versión 11 de aplicaciones estadísticas.

\section{RESULTADOS}

La edad media de nuestros pacientes en el momento del diagnóstico fue de 71,95 \pm 8,23 años (53-90). El tiempo de seguimiento de estos desde el diagnóstico del cáncer de próstata fue de una mediana de 24 meses (3-28). En cuanto al estadio tumoral en el momento del diagnóstico, 4 $(10,8 \%)$ pacientes presentaron un estadio pTlc, 22 (59,5\%) estadio pT2, y 11 (28,7\%) un pT3. Respecto a la presencia de enfermedad a distancia al diagnóstico, 6 pacientes presentaron adenopatías, y 37 metástasis óseas (Fig. 1).

Respecto a los pacientes que presentaron metástasis óseas en el momento del diagnóstico 21 de ellos presentaban más de 7 focos metastáticos mientras que 12 presentaban menos de 7 .

El grado de Gleason fue determinado en 26 pacientes mediante biopsias transrectales en el momento del diagnóstico, presentando, 9 de ellos un escore de Gleason 6, 12 un Gleason 7,4 un Gleason 8 y en 1 caso el escore era mayor de 8 . En 11 pacientes debido a los niveles de PSA y al tacto rectal sospechoso, se decidió iniciar tratamiento sin diagnóstico histológico (Fig. 2).

La mediana del PSA inicial fue $130 \mathrm{ng} / \mathrm{ml}(4,1-$ 100000), siendo la media de 3248,63 ng/ml. En 19 pacientes $(51,3 \%)$ los niveles del PSA en el momento del diagnóstico fueron mayores de $100 \mathrm{ng} / \mathrm{ml}$.

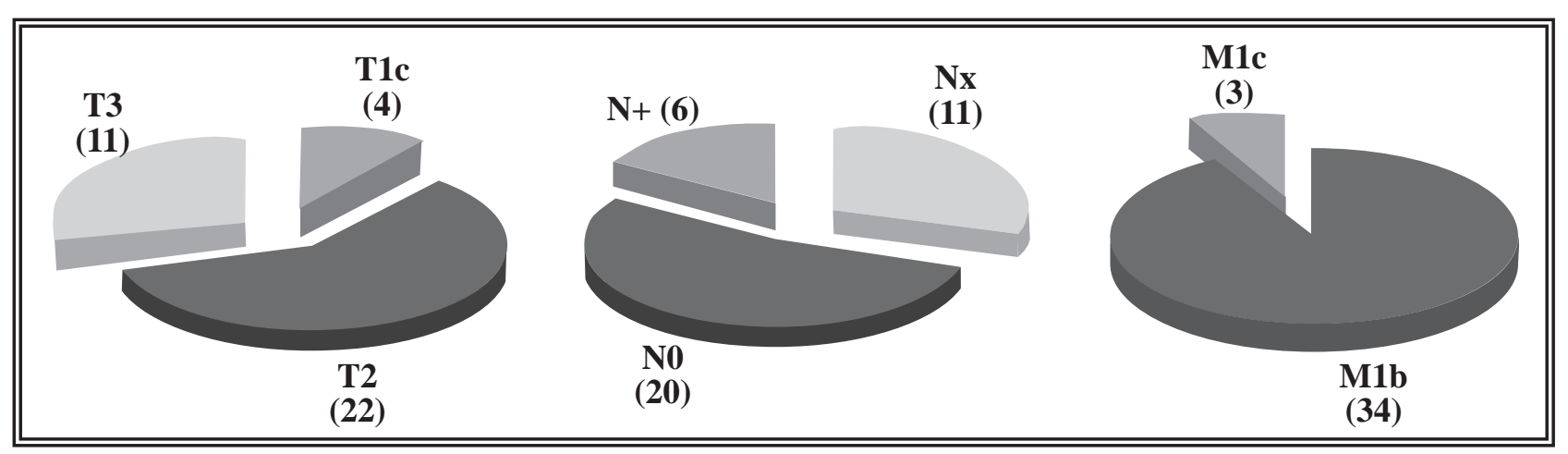

FIGURA 1. Estadio al diagnóstico 


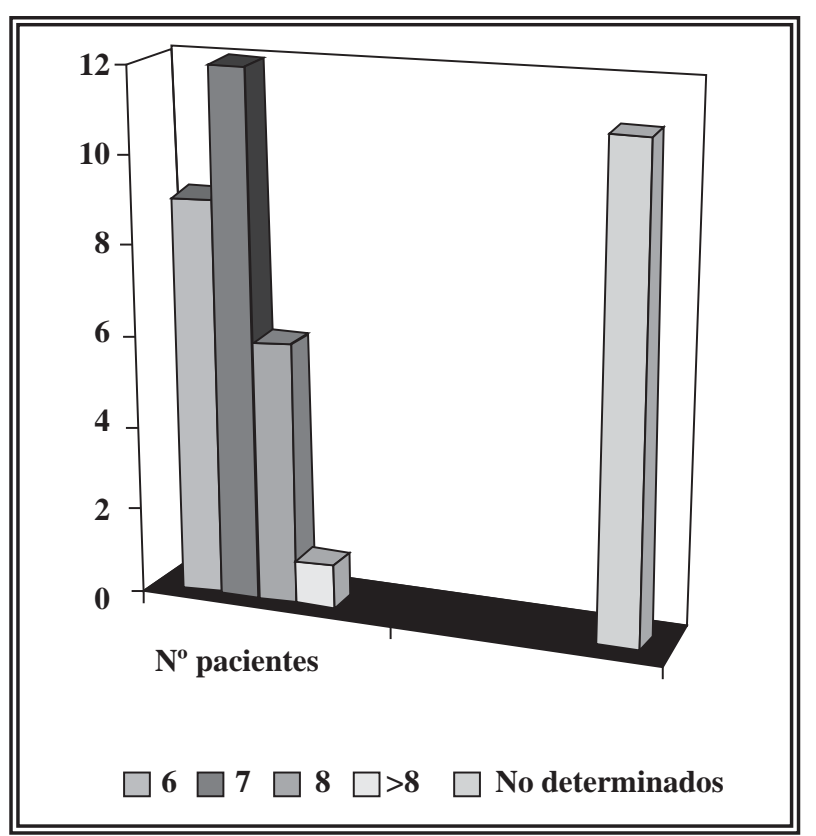

FIGURA 2. Score de Gleason en el momento del diagnóstico.

Respecto al tratamiento inicial en estos pacientes, 32 fueron sometidos a hormonoterapia y 5 a prostatectomía radical (en estos 5 casos todos presentaban en el momento del diagnóstico un $\mathrm{PSA} \leq 10,2 \mathrm{ng} / \mathrm{ml}$, y un score de Gleason $\leq 7$ (sólo 1 caso presentaba un escore de 7).

Se encontró incremento del PSA en 23 casos tras hormonoterapia. En 4 casos tras prostatectomía radical se empleó hormonoterapia por recidiva bioquímica y se demostró hormonorresistencia posterior, siendo necesario tratamiento de $2^{\mathrm{a}}$ línea (3 casos de estos presentaban metástasis óseas). $\mathrm{El}$ otro paciente sometido a prostatectomía radical mostró recidiva sin hormonorresistencia.

La mediana de meses en los que apareció hormonorresistencia desde el diagnóstico fue de 24 meses (4-112). Además la mediana de seguimiento posterior en estos pacientes fue de 8 meses. Respecto a la presencia de dolor óseo, 20 pacientes presentaron dolor óseo en el momento del diagnóstico, empleándose diferentes tratamientos de inicio y posteriormente, de acuerdo con la intensidad del dolor y localización (aunque con mala respuesta a estos): AINEs y mórficos, radioterapia localizada en focos bien delimitados (n: 2) y radioisótopos (Samario) en 7 casos.

En 20 pacientes se inicio el tratamiento con Zometa por dolor motivado por las metástasis óseas, mientras que 17 pacientes presentaban metástasis óseas, pero se encontraban asintomáticos. La mediana de seguimiento tras la instauración de Zometa fue de 11 meses (2-21), administrándose una media de 10,24 $\pm 5,42$ tratamientos. La media de PSA tras los 11 meses de seguimiento fue de $316,57 \pm 1118,3 \mathrm{ng} / \mathrm{ml}$ (mediana: $8,3 ; 0,1-6255)$.

Respecto a los efectos adversos del Zometa, no observamos ningún efecto secundario en ninguno de nuestros pacientes ni durante la inyección del fármaco ni posteriormente, si bien en 2 pacientes se suspendió el tratamiento por su mal estado general. Tampoco se registró ningún evento relacionado con el esqueleto.

El estado de los pacientes en el momento de finalizar el estudio fue de 5 pacientes fallecidos por el tumor, 22 pacientes vivos con enfermedad estable, y 10 pacientes vivos pero con enfermedad progresiva (Fig. 3).

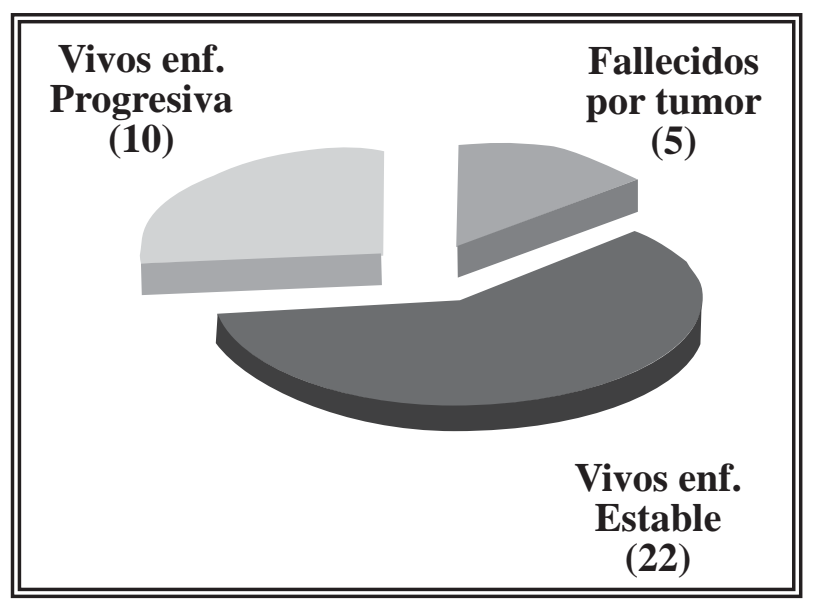

FIGURA 3. Estado de los pacientes al final del seguimiento.

En cuanto al control del dolor, observamos como en 8 de los 20 pacientes a los que se les administró Zometa, el control fue absoluto, en 6 de estos se consiguió un control parcialmente bueno (permitiendo una buena actividad diaria), en 4 el control fue parcialmente malo (empeorando su calidad de vida), mientras que 2 pacientes presentaron mal control del dolor (Fig. 4).

\section{DISCUSIÓN Y CONCLUSIONES}

El cáncer de próstata (CP) representa el tumor urológico más frecuente en varones. Se ha descrito como la cuarta neoplasia en todo el mundo 


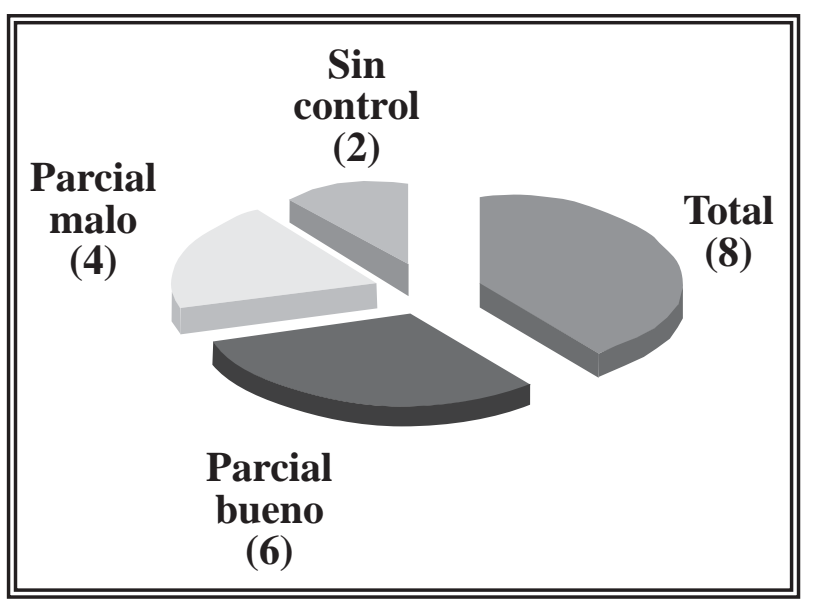

FIGURA 4. Respuesta al dolor tras tratamiento.

y la tercera en el varón, por detrás del melanoma y del cáncer de pulmón. En la actualidad es la neoplasia visceral más frecuente en EEUU y ha desbancado al cáncer de pulmón como primera causa de muerte por tumor debido a las campañas antitabaco ${ }^{11}$. En Europa se ha estimado que el $\mathrm{CP}$ es el tumor maligno más frecuente que afecta al hombre, siendo la segunda causa de muerte por cáncer, después del pulmonar.

El comportamiento del cáncer de próstata es muy heterogéneo abarcando un amplio espectro de situaciones clínicas. Puede presentarse como enfermedad diseminada, al diagnóstico, sin síntomas de crecimiento local. En otras ocasiones los síntomas por extensión locorregional preceden a la enfermedad a distancia o se presentan de forma asociada, aunque en ocasiones puede existir masa tumoral con compromiso de las estructuras adyacentes y sin enfermedad metastática acompañante, pero estas situaciones son una excepción, y lo habitual es que grandes masas tumorales presenten enfermedad a distancia.

El dolor originado por un proceso tumoral, puede controlarse eficazmente a través de medios relativamente sencillos hasta en un $90 \%$ de pacientes con cáncer o antecedentes de cáncer. Desafortunadamente, el dolor relacionado con el cáncer no suele ser tratado muy eficazmente ${ }^{9}$. Aunque no siempre se puede eliminar por completo el dolor tumoral o los sintomas que lo acompañan, el tratamiento del dolor mejora la calidad de vida del paciente durante todos los estadios de la enfermedad.
En nuestro caso quisimos comprobar su eficacia sobre el control del dolor originado por metástasis óseas, así como su perfil de seguridad. Observamos como en el $40 \%$ de los pacientes (n:8) el control fue completo, mientras que en el $30 \%$ (n:6) el control fue parcial, encontrando esta mejoría a partir del $2^{\circ}-3^{\text {er }}$ ciclo en 12 pacientes (60\%). Respecto a la tolerancia del fármaco ésta fue buena, no presentando ningún efecto secundario ni durante la administración ni posteriormente, suspendiendo sólo el tratamiento en 2 pacientes debido a su mal estado general. Tampoco observamos ningún evento relacionado con el esqueleto en ninguno de nuestros pacientes. Estos hechos corroboran los llevados a cabo por Saad et al. ${ }^{12}$ quienes observaron que el ácido zoledrónico es efectivo en prevenir y retrasar la aparición de eventos relacionados con el esqueleto en pacientes con metástasis óseas y tumores genitourinarios, (no sólo cáncer de próstata).

El futuro de este tratamiento reside en saber si la administración precoz de este fármaco puede prevenir o retrasar la aparición de las metástasis, (merced a su actividad antitumoral demostrada), no sólo en las debidas al cáncer de próstata si no también a metástasis óseas secundarias a otros tumores genito-urinarios, así como la osteoporosis inducida por la hormonoterapia. Para ello se están llevando a cabo numerosos estudios como el del Scandinavian Prostate Cancer Group de los que se esperan resultados próximamente.

\section{REFERENCIAS}

1. Ferlay J, Bray F, Pissani P, Parkin DM. Globocan 2000. Cancer incidence, mortality and prevalence worldwide, version 1.0. LARC Cancerbase no. 5. Lyon: IARCPress: 2001.

2. Luján Galán M, Páez Borda A, Chiva Robles V, Santonja Garriga C, Romero Cajigal I, Berenguer Sánchez A. Tendencias epidemiológicas en el cáncer de próstata durante los últimos años. Arch Esp Urol. 2004;57(8):817-825.

2. Luján Galán M, Páez Borda A, Chiva Robles V, Santonja Garriga C, Romero Cajigal I, Berenguer Sánchez A. Epidemiological trends in prostate cancer over the last years. Arch Esp Urol. 2004;57(8):817-825.

3. Hass GP, Sakr WA. Epidemiology of prostate cancer. CA Cancer J Clin. 1997;47(5):273-285.

4. Carlin BI, Andriole GL. The natural history, skeletal complications, and management of bone metastases in patients with prostate carcinoma. Cancer. 2000;88(12 Suppl):29892994. 
5. Berruti A, Dogliotti L, Bitossi R, Fasolis G, Gorzegno G, Bellina $\mathrm{M}$, et al. Incidence of skeletal complications in patients with bone metastatic prostate cancer and hormone refractory disease: predictive role of bone resorption and formation markers evaluated at baseline. $J$ Urol. 2000;164 (4): 1248-1253.

6. Saad F, Gleason DM, Murray R, Tchekmedyian S, Venner $\mathrm{P}$, Lacombe L, et al. Long term efficacy of zoledromic acid for the prevention of skeletal complications in patients with metastatic hormone-refractory prostate cancer. J Natl Cancer Inst. 2004;96(11):879-882.

7. Weinfurt KP, Li Y, Castel LJ, Timbie JW, Glendenning A Schulman KA. The impact of skeletal-related events on health related quality of life on patients with metastatic prostate cancer. Ann Oncol. 2002;13(suppl 5):180.

8. Riggs BL, Melton LJ 3rd. The worldwide problem of osteoporosis: insights afforded by epidemiology. Bone. 1995;17 (5 Suppl):505S-511S.

9. Weiss SC, Emanuel LL, Fairclough DL, Emanuel EJ. Understanding the experience of pain in terminally ill patients. Lancet. 2001;357(9265):1311-1315.
10. Meuser T, Pietruck C, Radbruch L, Stute P, Lehmann KA, Grond S. Symptoms during cancer pain treatment following WHO-guidelines: a longitudinal follow-up study of symptom prevalence, severity and etiology. Pain. 2001;93 (3):247-257.

11. Landis SH, Murria J, Bolden S. Cancer statistics 1999. CA Cancer J Clin 1999;49(1):8-31.

12. Saad F, Lipton A. Zoledronic acid is effective in preventing and delaying skeletal events in patients with bone metastases secondary to genitourinary cancers. BJU Int. 2005; 96(7):964-969.

Correspondencia autor: Dr. M. Álvarez-Múgica

Servicio de Urología 1

Hospital Universitario Central de Asturias

Celestino Villamil, s/n - 33006-Oviedo (Asturias)

Tel.: 985108000 (extensión 38143)

E-mail autor: malvarezmug@gmail.com

Información artículo: Original - Cáncer de próstata

Trabajo recibido: marzo 2007

Trabajo aceptado: junio 2007 